\title{
Ein starker Bankensektor - Fluch oder Segen?
}

\section{Hannah Winterberg}

\section{Relevanz}

Das Finanzsystem muss die unterschiedlichsten Bedürfnisse erfüllen. Unternehmen und Haushalte brauchen Eigenmittel und Fremdkapital in der richtigen Mischung. Eigenkapital ist die Voraussetzung für neue Kredite, denn überschuldeten Unternehmen und Haushalten mit geringen Eigenmitteln gibt ein vorsichtiger Banker keine weiteren Kredite. Um das Wachstum zu erhalten, sollten Unternehmen vordringlich Eigenkapital aufnehmen anstatt mit geringerer Kreditaufnahme ihre Investitionen einzuschränken. Wenn Bankkredite knapper werden, weil sich auch Banken entschulden müssen und weniger Kredite refinanzieren können, brauchen Unternehmen einen alternativen Weg der Fremdfinanzierung über Anleihenmärkte. Damit das Finanzsystem Wachstum und Wohlfahrt fördern kann, kommt es eben auf die richtige Mischung und Arbeitsteilung zwischen Banken, Börsen und anderen Finanzmarktakteuren an.

Christian Keuschnigg

\section{Quelle}

Der nachfolgende Text ist eine Zusammenfassung von: Langfield, Sam und Marco Pagano (2014), Bank Bias in Europe: Effects on Systemic Risk and Growth, Economic Policy, 2016, vol. 31, issue 85, 51-106.

H. Winterberg $(\bowtie)$

Universität St. Gallen, St. Gallen, Schweiz

E-Mail: hannah.winterberg@student.unisg.ch

(C) Der/die Autor(en) 2018

C. Keuschnigg (Hrsg.), Inklusives Wachstum und wirtschaftliche Sicherheit, https://doi.org/10.1007/978-3-658-21344-2_23 
Die Finanzsysteme Europas und der USA unterscheiden sich ganz erheblich, insbesondere in der den Banken zukommenden Rolle. Das angelsächsische System ist stärker kapitalmarktorientiert. Das bedeutet, dass Unternehmen ihren Finanzbedarf häufig am Kapitalmarkt decken, beispielsweise indem sie Anleihen ausgeben, und nur selten auf Kredite zurückgreifen. Im Gegensatz dazu ist das europäische Finanzsystem eher bankenorientiert. Es ist üblich, dass sich Unternehmen über Bankkredite refinanzieren.

Das europäische Finanzsystem wird von Banken dominiert. Die Bilanzsummen der Banken sind im Verhältnis zur Größe des Kapitalmarkts um ein Vielfaches grösser als in den USA.

Beide Systeme haben ihre Vor- und Nachteile. Banken mildern die Informationsnachteile der Kreditgeber gegenüber den Gläubigern, indem sie eine Beziehung zu ihren Kunden aufbauen. Sie ermöglichen den Kunden die leichtere Geheimhaltung von Geschäftsgeheimnissen, da weniger Informationen veröffentlicht werden müssen. Oft bewilligt nur die Hausbank einen Kredit, weil sie wesentlich besser über das Unternehmen Bescheid weiß als andere mögliche Kapitalgeber. Das macht viele Unternehmen in ihrer Kreditfinanzierung von der Hausbank abhängig und gibt ihr eine starke Verhandlungsposition. Deshalb sind die Zinskosten in einem bankorientierten System tendenziell höher. In einem marktorientierten System ist angesichts guter Alternativen die Verhandlungsposition der Kreditgeber schwächer, sodass die Gläubiger bessere Konditionen erzielen können. Allerdings erfordert der direkte Zugang zum Kapitalmarkt die Veröffentlichung von Firmendaten und ist für kleinere Unternehmen mit Aufwand verbunden. Die Literatur beleuchtet verschiedene Aspekte von kapitalmarkt- und bankorientierten Systemen, kommt aber nicht zu einer klaren Empfehlung, welches System zu bevorzugen ist.

Die unterschiedlichen Ausrichtungen spiegeln sich in der Kapitalisierung der Bankensysteme wieder. In Europa verfügen die Banken über Aktiva im Wert von 42 Billionen $€$, dies entspricht $334 \%$ des BIPs. Im Vergleich hat die EU damit den größten Bankensektor. Japanische Banken besitzen Aktiva im Wert von 8 Billionen $€$ oder $196 \%$ des BIPs, amerikanische im Wert von 14,5 Billionen $€$ oder $115 \%$ des BIPs. Diese beeindruckenden Unterschiede in der Kapitalisierung des Bankensektors haben sich vor allem in den letzten zwei Jahrzehnten herausgebildet, in denen der europäische Bankensektor sehr schnell gewachsen ist. Diese Entwicklung sieht man nicht nur in der absoluten Kapitalisierung des Bankensektors. Auch im Verhältnis zum privaten Aktien- und Anleihenmarkt ist der Bankensektor drastisch gewachsen. 
Die neue Studie von Langfield und Pagano untersucht den Einfluss dieser Abhängigkeit von Bankkrediten auf das systemische Risiko im Bankenmarkt und auf das Wirtschaftswachstum eines Landes. Die Forscher stellen fest, dass das Volumen der vergebenen Bankkredite volatil ist und sich stark prozyklisch entwickelt. Die Finanzierung über den Bankensektor verhält sich somit deutlich zyklischer als diejenige über Anleihenmärkte. Dies widerspricht der intuitiven Vermutung, dass eine enge Bank-Kunden-Beziehung die Kreditvergabe weniger von der wirtschaftlichen Entwicklung abhängig macht. Allerdings führt ein hoher Verschuldungsgrad dazu, dass Banken Schulden abbauen müssen, wenn die Preise von Vermögenswerten fallen, und damit weniger Kredite vergeben können. Wenn sie Verluste auf ihre Vermögenswerte erleiden, schmilzt das Eigenkapital und kann leicht unter die Mindestgrenze fallen. Ist neues Eigenkapital nicht verfügbar, müssen sie Vermögenswerte veräußern, um aus den Erlösen ihre Schulden zurückzuzahlen und auf diesem Weg die Mindestkapitalvorschriften zu erfüllen. Zum anderen werden viele Vermögenswerte als Sicherheiten genutzt. Verlieren sie an Wert, müssen die Banken neue Sicherheiten bereitstellen oder Schulden abbauen. Durch die Veräußerung von Vermögenswerten erhöht sich der Preisdruck zusätzlich und treibt den beschriebenen Prozess weiter an. Somit ist ein bankenorientiertes Finanzsystem nicht nur anfällig für Preisschwankungen von Vermögenswerten, sondern verstärkt diese sogar.

Im Vergleich zu kapitalmarktorientierten Systemen führt die Bankenorientierung zu höheren systemischen Risiken und zu geringerem Wirtschaftswachstum.

Bankenorientierte Finanzsysteme weisen ein höheres systemisches Risiko auf als kapitalmarktorientierte Systeme, insbesonders wenn in einer Krise die Vermögenswerte stark fallen. Diese erste Hypothese begründen die Autoren mit den starken Schwankungen bei der Kreditvergabe. Diese führt dazu, dass in Zeiten des Aufschwungs zu viele Kredite vergeben werden, sodass auch Projekte finanziert werden, die unter normalen Bedingungen nicht rentabel sind. Die vermehrte Übernahme von Risiken steigert in einer späteren Rezession die Ansteckungsgefahr und damit das systemische Risiko.

Diese Hypothese wird mit einer ökonometrischen Analyse von Daten von über 500 Banken im Zeitraum von 2000 bis 2012 bestätigt. Die Forscher weisen nach, dass das systemische Risiko steigt, wenn Länder ein höheres Bank-Markt-Verhältnis haben, also eine vergleichsweise größere Kapitalisierung im Bankensektor aufweisen. Das systemische Risiko messen sie hierbei mit der Menge an Eigenkapital, das eine Bank im Falle einer Immobilien- oder Börsenkrise aufbringen müsste. 
Ein Vergleich zwischen Deutschland mit einem Bank-Markt-Verhältnis von 5,7 und den USA mit einem Verhältnis von 0,7 im Jahr 2011 veranschaulicht die Unterschiede in den systemischen Risiken. Eine Bank in Deutschland mit Aktiva im Wert von 1 Bio. $€$ (die Bilanzsumme der Deutschen Bank beträgt z. B. 1,6 Bio. €, die der Erste Group Bank 0,2 Bio. $€$ ) würde im Falle einer Immobilienkrise ein systemisches Risiko in Höhe von 78 Mil. € darstellen. Dieselbe Bank würde jedoch in einem Land wie den USA mit einem weit niedrigeren Bank-Markt-Verhältnis lediglich ein systemisches Risiko in Höhe von 48 Mil. € darstellen.

Bankorientierte Finanzsysteme zeichnen sich durch ein niedrigeres Wachstum aus. Diese Wachstumseinbußen treten gerade dann ein, wenn Vermögenswerte stark fallen. Diese zweite Hypothese beruht auf einer ähnlichen Argumentationskette wie die erste, stellt allerdings einen anderen Aspekt in den Vordergrund. Wenn Banken in einer Rezession wenig Kredite vergeben, werden manche Projekte, obwohl sie rentabel wären, nicht finanziert. Vergeben Banken dagegen in einem Boom zu viele Kredite, werden auch Projekte finanziert, die unter normalen Bedingungen nicht mehr rentabel sind. Diese beiden Effekte führen zu einer ineffizienten Kapitalverwendung, welche das Wirtschaftswachstum dämpft.

Diese zweite Hypothese wird in der Studie ebenfalls empirisch verifiziert. Hierzu verwenden die Autoren Daten von rund 750 Banken aus dem Zeitraum 1988 bis 2011. Sie finden heraus, dass das Bank-Markt-Verhältnis negativ mit dem BIP-Wachstum korreliert ist. Das bedeutet, dass eine Erhöhung der Kapitalisierung im Bankensystem im Vergleich zum Aktien- und Anleihenmarkt in den folgenden Jahren ein niedrigeres Wachstum erwarten lässt, wenn andere Ursachen für Wachstumsunterschiede ausgeblendet werden.

Der Einfluss des Bank-Markt-Verhältnisses auf das systemische Risiko und die wirtschaftliche Entwicklung ist besonders bei Immobilienkrisen relevant, während bei einer Börsenkrise der Einfluss eher gering ist. Konkret bedeutet dies, dass das jährliche Wachstum in einem Land, dessen Bank-Markt-Verhältnis dem deutschen ähnelt, im Falle einer fünf Jahre andauernden Immobilienkrise um 3,6 Prozentpunkte stärker gehemmt wird, als das Wachstum eines Landes, dessen Finanzmarktstruktur den USA ähnelt. Dieser Unterschied erklärt sich durch die wichtige Rolle, die Immobilien als Sicherheiten bei der Kreditvergabe spielen.

Angesichts der ungünstigen Auswirkungen einer hohen Bankenorientierung stellt sich die Frage, wieso diese sich in Europa so stark herausgebildet hat. Das überdurchschnittliche Wachstum des Bankensektors hat hauptsächlich in den letzten zwei Jahrzehnten stattgefunden und ist ausschließlich auf das Wachstum der zwanzig größten Banken zurückzuführen. Diese Beobachtung lässt vermuten, dass staatliche Regulierungen diese Entwicklung begünstigt haben. Der Zwang zur staatlichen Stützung von Banken, welche zu groß zum Scheitern (Too-BigTo-Fail) sind, lässt Banken eine Vergrößerung Ihrer Bilanzsumme anstreben, da 
ihnen die implizite Staatsgarantie niedrigere Finanzierungskosten ermöglicht. Darüber hinaus neigen die europäischen Aufsichtsbehörden im Vergleich zu den amerikanischen dazu, Probleme von in Schwierigkeiten geratenen Banken eher durch Fusion als durch Abwicklung zu lösen.

In jüngster Vergangenheit gab es vier Gesetzesänderungen in der EU, welche dieser Entwicklung entgegenwirken und Banken stärker regulieren sollen. Dies sind erhöhte Kapitalvorschriften, eine zentralisierte Aufsichtsbehörde, eine neue Bankenabwicklungsrichtlinie (BRRD) sowie ein neuer einheitlicher Mechanismus zur Bankenabwicklung (SRM).

Die europäische Kommission plant mit der Errichtung einer Kapitalmarktunion der starken Bankenorientierung entgegenzuwirken und will die Möglichkeiten für eine marktorientierte Unternehmensfinanzierung verbessern.

Neben der stärkeren Regulierung von Banken hilft auch eine Weiterentwicklung des Wertpapiermarktes. Diese Weiterentwicklung ist ein Schlüsselvorhaben der Europäischen Kommission unter Präsident J.-C. Juncker, welche damit die bereits eingeführte Bankenunion durch eine Kapitalmarktunion komplettieren und damit den europäischen Unternehmen neue Finanzierungsmöglichkeiten eröffnen möchte. Die Studie empfiehlt in diesem Sinne, die weitere Integration der europäischen Börsen voranzutreiben, den Zugang zum Kapitalmarkt vor allem für kleine und mittlere Unternehmen zu erleichtern und die Entwicklung von Märkten für besicherte Unternehmensanleihen (sog. asset backed securities bzw. ABS-Märkten) zu fördern.

Open Access Dieses Kapitel wird unter der Creative Commons Namensnennung 4.0 International Lizenz (http://creativecommons.org/licenses/by/4.0/deed.de) veröffentlicht, welche die Nutzung, Vervielfältigung, Bearbeitung, Verbreitung und Wiedergabe in jeglichem Medium und Format erlaubt, sofern Sie den/die ursprünglichen Autor(en) und die Quelle ordnungsgemäß nennen, einen Link zur Creative Commons Lizenz beifügen und angeben, ob Änderungen vorgenommen wurden.

Die in diesem Kapitel enthaltenen Bilder und sonstiges Drittmaterial unterliegen ebenfalls der genannten Creative Commons Lizenz, sofern sich aus der Abbildungslegende nichts anderes ergibt. Sofern das betreffende Material nicht unter der genannten Creative Commons Lizenz steht und die betreffende Handlung nicht nach gesetzlichen Vorschriften erlaubt ist, ist für die oben aufgeführten Weiterverwendungen des Materials die Einwilligung des jeweiligen Rechteinhabers einzuholen.

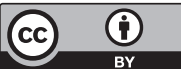

